\title{
A Fundamental Theorem on Initial Value Problems by using the Theory of Reproducing Kernels ${ }^{\dagger}$
}

\author{
L. P. Castro, M. M. Rodrigues and S. Saitoh
}

\begin{abstract}
We introduce a new method for solving general initial value problems by using the theory of reproducing kernels. The results are depending on the specific structure of each problem. Here, we give the general principle of the method and illustrate it with simple prototype examples. On the basis of the process, we have certain integral transforms, which are generated by each specific initial value problem, and need to be analysed. In view of this, we shall establish the basic relations among initial value problems for linear operator equations, eigenvalues and eigenfunctions in the related operator equations, integral transforms and associated reproducing kernels. Within this process, we will realize a general theory for operator equations and incorporate a time dependence in view to consider an associated regularization method.
\end{abstract}

Mathematics Subject Classification (2000). 58J47, 32A25, 45Q05, 46E22, 47A70, 47B32.

Keywords. Initial value problem, eigenfunction, eigenvalue, integral transform, integral equation, Aveiro discretization method, reproducing kernel, inverse problem.

\section{Introduction and general description}

It is well-known that analytical solutions of initial value problems are only known in somehow restrictive classes of such problems. This is why both numerical and theoretical methods are still highly welcome to be proposed and considered in the analysis of certain global classes of initial value problems.

\footnotetext{
$\dagger$ Accepted author's manuscript (AAM) published in [Complex Analysis and Operator Theory 9(1) (2015), 87-98] [DOI: 10.1007/s11785-014-0375-1] The final publication is available at Springer via http://dx.doi.org/10.1007/s11785-014-0375-1
} 
In the present paper we are just proposing a new global method of that kind. This will be highly based on the reproducing kernel theory, appropriate consequent spaces and a specific association to related eigenvalues and eigenfunctions. Namely, for some general linear operator $L_{x}$ (and differential operator $\partial_{t}$ ), for some function space on a certain domain (to be specified later on), we shall consider the problem

$$
\left(\partial_{t}+L_{x}\right) u_{f}(t, x)=0, \quad t>0
$$

for an unknown $u_{f}$ satisfying the initial value condition

$$
u_{f}(0, x)=f(x) .
$$

For such a very global problem, we shall propose a general method which includes the analysis of the existence and construction of the solution of that type of initial value problem by using the theory of reproducing kernels. Surprisingly enough, furthermore, our method will have the power to completely characterize the solutions under each specific conditions.

One of the basic procedures in our method is to use some eigenvalues $\lambda$ on a set $I$, and eigenfunctions $W_{\lambda}$ satisfying

$$
L_{x} W_{\lambda}(x)=\lambda W_{\lambda}(x) .
$$

The case of discrete eigenvalues may be dealt with similarly and so we shall assume that the eigenvalues are continuous on an interval $I$ for $\lambda>0$. In this way, we note that the functions

$$
\exp \{-\lambda t\} W_{\lambda}(x)
$$

are the solutions of the operator equation

$$
\left(\partial_{t}+L_{x}\right) u(t, x)=0 .
$$

We shall consider some general solution of (1.5) by a suitable sum of the elements in (1.4). In order to consider a convenient sum, we shall use the following kernel form, with a continuous non-negative weight function $\rho$ over the interval $I$,

$$
\int_{I} \exp \{-\lambda t\} W_{\lambda}(x) W_{\lambda}(y) \rho(\lambda) d \lambda
$$

(where we are naturally considering the integral with absolutely convergence for the kernel form). Moreover, here we assume $\lambda$ to be real-valued and also the eigenfunctions $W_{\lambda}(x)$ are real-valued. Then, fully general solutions of the equation (1.5) may be represented in the integral form

$$
u(t, x)=\int_{I} \exp \{-\lambda t\} W_{\lambda}(x) F(\lambda) \rho(\lambda) d \lambda .
$$

for the functions $F$ satisfying

$$
\int_{I} \exp \{-\lambda t\}|F(\lambda)|^{2} \rho(\lambda) d \lambda<\infty
$$

Thus, the solution $u(t, x)$ of (1.5) satisfying the initial condition

$$
u(0, x)=F(x)
$$


will be obtained by taking $t \rightarrow 0$ in (1.7). However, this point will be very delicate and we will need to consider some deep and intricate structure. Here, (1.6) is a reproducing kernel and in order to analyze in detail the strategy above, we will need the theory of reproducing kernels. In particular, in order to construct certain natural solutions of (1.6) we will need a new framework and function space.

\section{Preliminaries on linear mappings and inversions}

In order to analyze the integral transform (1.7) and in order to set the basic background for our purpose, we will need the essence of the theory of reproducing kernels.

We are interested in the integral transforms (1.7) in the framework of Hilbert spaces. This will strongly depend on the characterization of the image function spaces, an isometric identity (of Parseval type) and an inversion formula. For these general and fundamental problems, we have a unified fundamental method and concept within a quite global situation. Namely, having in mind [12, 13, 14, we shall recall a general theory for linear mappings in the framework of Hilbert spaces $\mathcal{H}$ (which may be finite-dimensional). For this purpose, let $E$ be an abstract set and $\mathbf{h}$ be a Hilbert $\mathcal{H}$-valued function on $E$. Then, we shall consider the linear transform

$$
f(x)=(\mathbf{f}, \mathbf{h}(x))_{\mathcal{H}}, \quad \mathbf{f} \in \mathcal{H},
$$

from $\mathcal{H}$ into the linear space $\mathcal{F}(E)$ comprising all the complex valued functions on $E$. In order to investigate the linear mapping (2.1), we consider a positive definite quadratic form function $K(x, y)$ on $E \times E$ defined by

$$
K(x, y)=(\mathbf{h}(y), \mathbf{h}(x))_{\mathcal{H}} \quad \text { on } \quad E \times E .
$$

We recall that a complex-valued function $k: E \times E \rightarrow \mathbb{C}$ is called a positive definite quadratic form function on the set $E$, or shortly, positive definite function, when it satisfies the property that, for an arbitrary function $X: E \rightarrow \mathbb{C}$ and any finite subset $F$ of $E$,

$$
\sum_{x, y \in F} \overline{X(x)} X(y) k(x, y) \geq 0 .
$$

By the fundamental theorem, we know that for any positive definite quadratic form function $K$, there exists a uniquely determined reproducing kernel Hilbert space admitting the reproducing property. This is in fact generating the following significant known properties.

Proposition 2.1 (cf. [6, 12, 13, 14]). (A) The range of the linear mapping (2.1) by $\mathcal{H}$ is characterized as the reproducing kernel Hilbert space $H_{K}(E)$ admitting the reproducing kernel $K(x, y)$ whose characterization is given by the two properties: (i) $K(\cdot, y) \in H_{K}(E)$ for any $y \in E$ and, (ii) for any $f \in H_{K}(E)$ and for any $x \in E,(f(\cdot), K(\cdot, x))_{H_{K}(E)}=f(x)$. 
(B) We have the inequality $\|f\|_{H_{K}(E)} \leq\|\mathbf{f}\|_{\mathcal{H}}$. Moreover, for any element $f$ of $H_{K}(E)$ there exists a uniquely determined $\mathbf{f}^{*} \in \mathcal{H}$ satisfying

$$
f(x)=\left(\mathbf{f}^{*}, \mathbf{h}(x)\right)_{\mathcal{H}} \quad \text { on } E
$$

and

$$
\|f\|_{H_{K}(E)}=\left\|\mathbf{f}^{*}\right\|_{\mathcal{H}} .
$$

(C) We have the inversion formula in (2.1) in the form

$$
f \mapsto \mathbf{f}^{*}
$$

in $(B)$ by using the reproducing kernel Hilbert space $H_{K}(E)$.

However, for the formula (2.3) we have no global form and so it is needed to apply here different case by case arguments. See [13] and [14 for some details and applications on this issue. Recently, however, we obtained a very general inversion formula based on the so-called Aveiro Discretization Method in Mathematics (cf. [3]) by using the ultimate realization of reproducing kernel Hilbert spaces.

In this paper, however, in order to give prototype examples with analytical nature, we shall consider the following general inversion formula in a global situation with certain natural assumptions. Namely, in order to derive a general inversion formula for a widely applicable situation in analysis, we assume that $\mathcal{H}=L^{2}(I, d m)$ and that $H_{K}(E)$ is a closed subspace of $L^{2}(E, d \mu)$. Furthermore, below we assume that $(I, \mathcal{I}, d m)$ and $(E, \mathcal{E}, d \mu)$ are both $\sigma$-finite measure spaces and that

$$
H_{K}(E) \hookrightarrow L^{2}(E, d \mu) .
$$

Suppose that we are given a measurable function $h: I \times E \rightarrow \mathbb{C}$ satisfying $h_{y}=h(\cdot, y) \in L^{2}(I, d m)$ for all $y \in E$. Let us set

$$
K(x, y) \equiv\left\langle h_{y}, h_{x}\right\rangle_{L^{2}(I, d m)} .
$$

As we have established in Proposition 2.1, we have

$$
H_{K}(E) \equiv\left\{f \in \mathcal{F}(E): f(x)=\left\langle F, h_{x}\right\rangle_{L^{2}(I, d m)} \text { for some } F \in \mathcal{H}\right\} .
$$

Let us now define

$$
L: \mathcal{H} \rightarrow H_{K}(E) \quad\left(\hookrightarrow L^{2}(E, d \mu)\right)
$$

by

$$
\begin{aligned}
L F(x) & \equiv\left\langle F, h_{x}\right\rangle_{L^{2}(I, d m)} \\
& =\int_{I} F(\lambda) \overline{h(\lambda, x)} d m(\lambda), \quad x \in E,
\end{aligned}
$$

for $F \in \mathcal{H}=L^{2}(I, d m)$, keeping in mind (2.4). Observe that $L F \in H_{K}(E)$.

The next result will serve to the inversion formula.

Proposition 2.2 (cf. [13]). Assume that $\left\{E_{N}\right\}_{N=1}^{\infty}$ is an increasing sequence of measurable subsets in $E$ such that

$$
\bigcup_{N=1}^{\infty} E_{N}=E
$$


and that

$$
\int_{I \times E_{N}}|h(\lambda, x)|^{2} d m(\lambda) d \mu(x)<\infty
$$

for all $N \in \mathbb{N}$. Then we have

$$
L^{*} f(\lambda)\left(=\lim _{N \rightarrow \infty}\left(L^{*}\left[\chi_{E_{N}} f\right]\right)(\lambda)\right)=\lim _{N \rightarrow \infty} \int_{E_{N}} f(x) h(\lambda, x) d \mu(x)
$$

for all $f \in L^{2}(I, d \mu)$ in the topology of $\mathcal{H}=L^{2}(I, d m)$. Here, $L^{*} f$ is the adjoint operator of $L$, but it represents the inversion with the minimum norm for $f \in$ $H_{K}(E)$.

In this Proposition 2.2, we see in a very natural way that the inversion formula may be given in the strong convergence in the space $\mathcal{H}=L^{2}(I, d m)$.

\section{Main result}

Having in mind the general framework of Section 2, we shall now built our general theorem. For this purpose, we will assume that $I$ is a positive interval, $\lambda>0$, and that this parameter $\lambda$ represents the eigenvalues satisfying

$$
L_{x} \overline{h(\lambda, x)}=\lambda \overline{h(\lambda, x)}, \quad x \in E, \quad \lambda \in I .
$$

Here, $\overline{h(\lambda, x)}$ is the eigenfunction and in order to set our notation in a consistent way, we put the complex conjugate there.

We form the reproducing kernel

$$
K_{t}(x, y)=\int_{I} \exp \{-\lambda t\} h(\lambda, y) \overline{h(\lambda, x)} d m(\lambda), \quad t>0,
$$

and consider the reproducing kernel Hilbert space $H_{K_{t}}$ admitting the kernel $K_{t}(x, y)$. In particular, note that

$$
K_{t}(x, y) \in H_{K}(E), \quad y \in E .
$$

Then, we have all the material to state and derive the following main theorem of this work.

Theorem 3.1. For any element $f \in H_{K}(E)$, the solution $u_{f}(t, x)$ of the initial value problem (1.1)-(1.2) exists and it is given by

$$
u_{f}(t, x)=\left(f(\cdot), K_{t}(\cdot, x)\right)_{H_{K}(E)} .
$$

Here, the meaning of the initial value (1.2) is given by

$$
\begin{aligned}
\lim _{t \rightarrow+0} u_{f}(t, x) & =\lim _{t \rightarrow+0}\left(f(\cdot), K_{t}(\cdot, x)\right)_{H_{K}(E)} \\
& =(f(\cdot), K(\cdot, x))_{H_{K}}(E) \\
& =f(x),
\end{aligned}
$$

whose existence is ensured and the limit is given in the sense of uniformly convergence on any subset of $E$ such that $K(x, x)$ is bounded. 
The uniqueness property of the initial value problem is depending on the completeness of the family of functions

$$
\left\{K_{t}(\cdot, x) ; x \in E\right\}
$$

in $H_{K}(E)$.

In our theorem, the complete property of the solutions $u_{f}(t, x)$ of (1.1)-(1.2) satisfying the initial value $f$ may be derived by the reproducing kernel Hilbert space admitting the kernel

$$
k(x, t ; y, \tau):=\left(K_{\tau}(\cdot, y), K_{t}(\cdot, x)\right)_{H_{K}(E)} .
$$

Some other more global observations are also already in order. In our method, we see that the existence problem of the initial value problem is based on the eigenfunctions and we are constructing the desired solution satisfying the considered initial condition. In view of this, for a larger knowledge for the eigenfunctions we can consider a more general initial value problem. Furthermore, by considering the linear mapping of (3.3) with various situations, we will be able to obtain various inverse problems which may be described by looking for the initial values $f$ from the various output data of $u_{f}(t, x)$.

We also would like to remark that in the stationary case of

$$
L_{x} u(x)=0,
$$

our method is more simple and direct. We may consider the family of solutions $\overline{h(\lambda, x)}$ such that

$$
L_{x} \overline{h(\lambda, x)}=0 .
$$

Then, the general solutions are constructed by the integral form

$$
u(x)=L F(x) \equiv\left\langle F, h_{x}\right\rangle_{L^{2}(I, d m)}=\int_{I} F(\lambda) \overline{h(\lambda, x)} d m(\lambda), \quad x \in E,
$$

for $F \in \mathcal{H}=L^{2}(I, d m)$.

Proof of Theorem 3.1. At first, note that the kernel $K_{t}(x, y)$ satisfies the operator equation (1.1) for any fixed $y$, because the functions

$$
\exp \{-\lambda t\} \overline{h(\lambda, x)}
$$

satisfy the operator equation and it is the summation. Similarly, the function $u_{f}(t, x)$ defined by (3.3) is the solution of the operator equation (1.1).

In order to see the initial value problem, we note the important general property:

$$
K_{t}(x, y) \ll K(x, y) ;
$$

that is, $K(x, y)-K_{t}(x, y)$ is a positive definite quadratic form function and we have

$$
H_{K_{t}} \subset H_{K}(E)
$$

and for any function $f \in H_{K_{t}}$, it holds

$$
\|f\|_{H_{K}(E)}=\lim _{t \rightarrow+0}\|f\|_{H_{K_{t}}}
$$


in the sense of non-decreasing norm convergence (cf. [1]). In order to verify the crucial point in (3.6), note that

$$
\begin{aligned}
\left\|K(x, y)-K_{t}(x, y)\right\|_{H_{K}(E)}^{2} & =K(y, y)-2 K_{t}(y, y)+\left\|K_{t}(x, y)\right\|_{H_{K}(E)}^{2} \\
& \leq K(y, y)-2 K_{t}(y, y)+\left\|K_{t}(x, y)\right\|_{H_{K_{t}}}^{2} \\
& =K(y, y)-K_{t}(y, y),
\end{aligned}
$$

that converges to zero as $t \rightarrow+0$. We thus obtain the desired limit property in the theorem. (3.3).

The uniqueness property of the initial value problem follows directly from

\section{Special cases}

\subsection{The derivative operator and the corresponding exponential function case}

For the simplest derivative operator $D$, we obtain, of course,

$$
D e^{\lambda x}=\lambda e^{\lambda x}
$$

We will be able to see that we can consider initial value problems with various situations by considering consequent $\lambda$ and the variable $x$. As typical cases we can point out the weighted Laplace transforms, the Paley-Wiener spaces and the Sobolev spaces depending on $\lambda>0, \lambda$ being on a symmetric interval or $\lambda$ on the whole real space.

The Laplace transform may be taken into account in many situations by considering various weights (see [13]), and so we shall consider the simplest case:

$$
K(z, \bar{u})=\int_{0}^{\infty} e^{-\lambda z} e^{-\lambda \bar{u}} d \lambda=\frac{1}{z+\bar{u}}, \quad z=x+i y,
$$

on the right half complex plane. The reproducing kernel is the Szegö kernel and we have the image of the integral transform

$$
f(z)=\int_{0}^{\infty} e^{-\lambda z} F(\lambda) d \lambda
$$

for the $L_{2}(0, \infty)$ functions $F(\lambda)$. Thus, we obtain the isometric identity

$$
\frac{1}{2 \pi} \int_{-\infty}^{+\infty}|f(i y)|^{2} d y=\int_{0}^{\infty}|F(\lambda)|^{2} d \lambda .
$$

Here, $f(i y)$ mean the Fatou's non-tangential boundary values of the Szegö space of analytic functions on the right hand half complex plane.

Now, we shall consider the reproducing kernel $K_{t}(z, \bar{u})$ and the corresponding reproducing kernel Hilbert space $H_{K_{t}}$ by taking

$$
K_{t}(z, \bar{u})=\int_{0}^{\infty} e^{-\lambda t} e^{-\lambda z} e^{-\lambda \bar{u}} d \lambda .
$$


Note that the reproducing kernel Hilbert space $H_{K_{t}}$ is the Szegö space on the right hand complex plane $x>\frac{-t}{2}$.

For any Szegö kernel function space member $f(z)$ on the right half complex plane, the function

$$
\begin{aligned}
U_{f}(t, z) & =\left(f(\cdot), K_{t}(\cdot, \bar{z})_{H_{K}}\right. \\
& =\frac{1}{2 \pi} \int_{-\infty}^{+\infty} f(i y) \overline{K_{t}(i y, \bar{z})} d y
\end{aligned}
$$

satisfies the partial differential equation

$$
\left(\partial_{t}-D_{z}\right) U(t, z)=0 \text {. }
$$

For the sake of the monotonicity of the reproducing kernels, it holds

$$
K_{t}(z, \bar{u}) \ll K(z, \bar{u}) ;
$$

we obtain the desired initial condition:

$$
\begin{aligned}
\lim _{t \rightarrow 0} U_{f}(t, z) & =\lim _{t \rightarrow 0}\left(f(\cdot), K_{t}(\cdot, \bar{z})\right)_{H_{K}} \\
& =(f(\cdot), K(\cdot, \bar{z}))_{H_{K}} \\
& =f(z) .
\end{aligned}
$$

From the general property of the reproducing kernels, we see that the above convergence is uniform on any compact subset of the right half complex plane.

In order to see the characteristic property of the solutions $U(t, z)$, we shall consider the kernel form

$$
\begin{aligned}
k(t, z ; \tau, \bar{u}) & =\left(K_{\tau}(\cdot, \bar{u}), K_{t}(\cdot, \bar{z})\right)_{H_{K}} \\
& =\frac{1}{t+\tau+z+\bar{u}} .
\end{aligned}
$$

From this representation we see that, for any fixed $t>0$, the solutions $U(t, z)$ belong to the Szegö space on the right hand complex plane

$$
\text { Re } z>-t \text {, }
$$

and for any fixed $z, R e z>0$, the solutions $U_{f}(t, z)$ may be continued analytically onto the half complex plane with respect to $t$,

$$
\operatorname{Re} t>-\operatorname{Re} z \text {, }
$$

In particular, note that the solutions $U_{f}(t, z)$ can be also interpreted for "negative time".

For any fixed time $t$, we can obtain the inversion formula in the complex version in (4.8) by our general formula in Proposition 2.2, because the needed situations are concretely given. Meanwhile, for any fixed space point $z$, we will be able to see that the situation is similar, and we naturally consider the inversion with the "complex time" $t$. When we wish to establish the real inversion, we can consider the inversion formulas by the Aveiro Discretization Method in Mathematics 3, 4, or by applying the Tikhonov Regularization Method 14 as in the numerical real 
inversion formula of the Laplace transform or in other concrete applications [2, 5, Then, the analytical inversion formula is very deep and complicated (cf. e.g. [13] for the real inversion formula of the Laplace transform). For very general situations with weighted Laplace transforms, the Paley-Wiener spaces and the Sobolev spaces, we would like to refer the reader to [13.

4.2. The case of the operator $L(D)=D^{2}$

In the case of $L(D)=D^{2}$, we have

$$
\sin \lambda x, \cos \lambda x .
$$

as eigenfunctions with $\lambda$ eigenvalues. So, we can consider the sine and cosine transforms with suitable weights on the positive real line (see, for example, [13]).

On the half line, we have the following important reproducing kernels:

$$
\begin{aligned}
K(s, t) & \equiv \int_{0}^{\infty} \frac{\cos (s \lambda) \cos (t \lambda)}{\lambda^{2}+1} d \lambda \\
& =\frac{\pi}{4}(\exp (-|s-t|)-\exp (-s-t))
\end{aligned}
$$

for $s, t>0$. Then we have

$$
H_{K}(0, \infty)=W^{1,2}(0, \infty)
$$

as a set and the norm is given by

$$
\|f\|_{H_{K}(0, \infty)}=\sqrt{\frac{2}{\pi} \int_{0}^{\infty}\left(\left|f^{\prime}(\lambda)\right|^{2}+|f(\lambda)|^{2}\right) d \lambda .}
$$

Meanwhile, in the sine case, let

$$
\begin{aligned}
K(s, t) & \equiv \int_{0}^{\infty} \frac{\sin (s \lambda) \sin (t \lambda)}{\lambda^{2}+1} d \lambda \\
& =\frac{\pi}{4}(\exp (-|s-t|)+\exp (-s-t))
\end{aligned}
$$

for $s, t>0$. Then we have

$$
H_{K}(0, \infty)=\left\{f \in W^{1,2}(0, \infty): f(0)=0\right\}
$$

as a set and the norm $\|\cdot\|_{H_{K}(0, \infty)}$ is still given by (4.12).

In addition, for the hyperbolic sine and cosine functions, we note the beautiful formulas

$$
\int_{0}^{\infty} \frac{\sinh a x \sinh b x}{\cosh c x} d x=\frac{\pi}{c} \frac{\sin \frac{a \pi}{2 c} \sin \frac{b \pi}{2 c}}{\cos \frac{a \pi}{c}+\cos \frac{b \pi}{c}}, \quad c>|a|+|b|,
$$

and

$$
\int_{0}^{\infty} \frac{\cosh a x \cosh b x}{\cosh c x} d x=\frac{\pi}{c} \frac{\cos \frac{a \pi}{2 c} \cos \frac{b \pi}{2 c}}{\cos \frac{a \pi}{c}+\cos \frac{b \pi}{c}}, \quad c>|a|+|b|
$$

(see 7], p. 372).

From these integral forms, we can examine the integral transforms, analytically. However, their detailed are intended to be done in a separate paper specially 
devoted to these cases. We would like to point out that these integral transform problems were proposed by Professor N. M. Tuan, incidentally, when discussing another problem, during his last visit to CIDMA - Center for Research and Development in Mathematics and Applications, University of Aveiro.

As a concluding remark and final suggestion, we stress that from the great books [7, 8, 9, 10, 11] one can find many concrete problems among partial differential equations, eigenfunctions, integral transforms and reproducing kernels to which our present method can be applied. In view of this, in order to obtain some analytical results for concrete cases, we have to analyze and realize the corresponding reproducing kernel Hilbert spaces. However, for this part of the process, in most of the cases, we can also take profit of the Aveiro Discretization Method in Mathematics [3, 4.

\section{Acknowledgments}

The authors were supported in part by Portuguese funds through the CIDMACenter for Research and Development in Mathematics and Applications (University of Aveiro) and the Portuguese Foundation for Science and Technology ("FCTFundação para a Ciência e a Tecnologia") within project UID/MAT/04106/2013. The third author is supported in part by the Grant-in-Aid for the Scientific Research (C)(2)(No. 24540113).

\section{References}

[1] Aronszajn, N.: Theory of reproducing kernels. Trans. Amer. Math. Soc. 68, 337-404 (1950)

[2] Castro, L.P., Chen, Q., Saitoh, Q.: Source inversion of heat conduction from a finite number of observation data. Appl. Anal. 89, 801-813 (2010)

[3] Castro, L.P., Fujiwara, H., Rodrigues, M.M., Saitoh, S., Tuan, V.K.: Aveiro Discretization Method in Mathematics: A New Discretization Principle. Mathematics without Boundaries: Surveys in Pure Mathematics, Panos Pardalos and Themistocles M. Rassias (edts.), 52 pp. Springer-Verlag, New York (to appear)

[4] Castro, L.P., Fujiwara, H., Qian, T., Saitoh, S.: How to catch smoothing properties and analyticity of functions by computers?. Mathematics without Boundaries: Surveys in Pure Mathematics, Panos Pardalos and Themistocles M. Rassias (edts.), 15 pp. Springer-Verlag, New York (to appear)

[5] Castro, L.P., Itou, H., Saitoh, S.: Numerical solutions of linear singular integral equations by means of Tikhonov regularization and reproducing kernels. Houston Journal of Mathematics 38(4), 1261-1276 (2012)

[6] Castro, L.P., Saitoh, S.: Optimal and approximate solutions of singular integral equations by means of reproducing kernels. Complex Anal. Oper. Theory 7, 1839-1851 (2013)

[7] Gradshlein, I.S., Ryzhik, I.M.: Table of Integrals, Series, and Products. Elsevier/Academic Press, Amsterdam (2007) 
[8] Prudnikov, A.P., Brychkov, Yu.A., Marichev, O.I.: Integrals and Series, Volume 3: More Special Functions. Gordon and Breach Publisher, New York (1990)

[9] Polyanin, A.D.: Handbook of Linear Partial Differential Equations for Engineers and Scientists. Chapman \& Hall/CRC, Boca Raton, FL (2002)

[10] Polyanin, A.D., Zaitsev, V.F.: Handbook of Exact Solutions for Ordinary Differential Equations. CRC Press, Boca Raton, FL (2003)

[11] Polyanin, A.D., Manzhirov, A.V.: Handbook of Integral Equations. CRC Press, Boca Raton, FL (2008)

[12] Saitoh, S.: Hilbert spaces induced by Hilbert space valued functions. Proc. Amer. Math. Soc. 89, 74-78 (1983)

[13] Saitoh, S.: Integral Transforms, Reproducing Kernels and their Applications. Pitman Research Notes in Mathematics Series 369, Addison Wesley Longman, Harlow (1997)

[14] Saitoh, S.: Theory of reproducing kernels: Applications to approximate solutions of bounded linear operator functions on Hilbert spaces. Amer. Math. Soc. Transl. Ser. 230, 107-134 (2010)

L. P. Castro

Center for Research and Development in Mathematics and Applications

Department of Mathematics, University of Aveiro

3810-193 Aveiro, Portugal

e-mail: castro@ua.pt

M. M. Rodrigues

Center for Research and Development in Mathematics and Applications

Department of Mathematics, University of Aveiro

3810-193 Aveiro, Portugal

e-mail: mrodrigues@ua.pt

S. Saitoh

Center for Research and Development in Mathematics and Applications

Department of Mathematics, University of Aveiro

3810-193 Aveiro, Portugal

e-mail: saburou.saitoh@gmail.com 